who experience life-threatening episodes of acute respiratory distress.

Results Fifty-four children were admitted on 72 occasions with wheeze. 32 children with asthma, 19 children with wheeze. Three children, admitted on 4 occasions, were excluded for alternative diagnoses. Unsurprisingly children with asthma were older (mean 92.54. sd 53.8 months) than children with wheeze (mean 24.79, sd 17.52 months).

Children with asthma were each admitted to PICU more frequently than children with wheeze (mean 1.97, sd 1.43 vs mean 1.26, sd 0.56). For the 68 included episodes, respiratory support was needed on 26 occasions (formal ventilation on 12).

In $49 \%$ of cases children lived in a household where a family member smoked (15 asthma; 5 wheeze). On 15 occasions household smoking status was not documented. In 16 cases of asthma there were documented social concerns (compliance, clinic attendance, formal safeguarding) compared to only 1 case of the 19 children with wheeze. Children in the asthma group came from larger families with 3.5 children (IQR 2,4 max 11) per family compared to 2.4 children (IQR 1,3 max 7) in the wheeze group.

Conclusion Children with the most severe episodes requiring critical care often present on multiple occasions and come from large families with household smoking exposure. Concerns regarding the inappropriately low priority of the children's health by their families are common.

\section{G457(P) INVESTIGATING THE EFFICACY OF HOME RESPIRATORY SURVEILLANCE IN THE MANAGEMENT OF RESPIRATORY CYSTIC FIBROSIS DISEASE IN PAEDIATRICS}

${ }^{1} \mathrm{Z}$ Childs Ford, ${ }^{2} \mathrm{~N}$ Rao. ${ }^{1}$ Manchester Medical School, University of Manchester, Manchester, UKi ${ }^{2}$ Paedatrics, Wythenshawe Hospital, Manchester, UK

\subsection{6/archdischild-2018-rcpch.446}

Background Cystic Fibrosis (CF) is an autosomal recessive condition in which much of the morbidity and mortality is attributed to the respiratory component of the disease. Microbiological surveillance of respiratory flora is vital in $\mathrm{CF}$ management. In addition to traditional clinic-based sampling, Wythenshawe hospital's paediatric CF department has been training parents to take cough swabs at home.

Aims To assess how effective home sampling is in the monitoring of respiratory flora of CF paediatric patients.

Methods Data on respiratory samples were collected for the year 01/01/2016-31/12/2016. Patients notes, electronic patient records and Lorenzo were accessed. Kappa testing was used to determine the level of agreement home and clinic samples.

Results 21 patients were identified for this study.

- A total of 172 home samples were collected

- On average, each patient had 8 home samples collected

- Absolute agreement between home and clinic samples was 91.92\%

- Number of home and clinic samples having excellent agreement was $10(52.63 \%)$

- There were several bacteria which were grown only in home or clinic samples. These bacteria are not commonly known to be contaminants

- On average home sampling lead to antibiotic treatment occurring 44.17 days before a clinic attendance
Conclusion The strong level of agreement between samples collected at home and in clinical settings suggests that the bacteria being detected at home is similar to what is detected in the clinics. The differences seen in bacteria grown in these two settings is likely to be a result of differences in the respiratory flora and not due to contamination of samples taken at home.

On average, home sampling resulted in antibiotic treatment 44.17 days before a clinic attendance would have occurred. This reduces the amount of time for inflammatory damage and antibiotic resistance to occur.

This study recommends the continuation of home sampling to boost sampling rates, thereby increasing the clinician's confidence in the each patient's microbiological profile.

Moving forward research should focus on gathering more information on the health benefits to patients receiving home sampling and on cost-benefit analysis.

\section{G458(P) ASSESSMENT OF THE SHORT-TERM CLINICAL OUTCOME OF CHILDREN WITH POLYARTICULAR AND SYSTEMIC ONSET JUVENILE IDIOPATHIC ARTHRITIS: A RETROSPECTIVE COHORT STUDY IN A TERTIARY CENTRE SAMPLE}

${ }^{1} \mathrm{~N}$ Elndari, ${ }^{2} \mathrm{~J}$ Camilleri, ${ }^{2} \mathrm{~J}$ Willock. 'School of Medicine, Cardiff University, Cardiff, UK; ${ }^{2}$ Rheumatology Department, University Hospital of Wales, Cardiff, UK

\subsection{6/archdischild-2018-rcpch.447}

Objectives To determine the early clinical improvement and short-term outcome of patients with polyarticular and systemic onset Juvenile Idiopathic Arthritis (JIA) achieved with current therapeutic agents and treatment strategies by analysing the early disease course and one-year outcome of a tertiary centre cohort of patients.

Methods All patients with a diagnosis of polyarticular or systemic onset JIA seen at a paediatric tertiary hospital were identified from the rheumatology clinic registry. Inclusion criteria was a time period of at least one year since the diagnosis. Patients were excluded if they were discharged or transferred to adults' service. Clinical records from the hospital database and medical charts were reviewed for data collection. The collected data was analysed using qualitative measures and results were drawn from observational findings. The outcomes reported in this study include: number of joints with active inflammation, systemic disease features, C-reactive protein (CRP), and functional ability of the child as recorded in the clinical letters.

Results A total of 45 patients were identified, of which 32 were eligible. The frequency of JIA subtypes: 19 patients with RF-negative polyarthritis, 7 with RF-positive polyarthritis and 6 with systemic onset disease. Out of the total, 9 (28\%) were completely asymptomatic and did not show any sign of active joint inflammation or residual joint changes at one year from diagnosis. Also at one year, 15 (47\%) had no signs of active disease on physical examination but musculoskeletal pain seems to persist in some. For functional ability, $81 \%$ did not have any limitation to their physical ability, while the rest $19 \%$ had variable degrees of ongoing restrictions to their mobility or activity.

Conclusion Significant improvement in disease activity was noted in all patients with the current therapeutic strategies in clinical practice. However, low levels of disease activity persisted in many 